\title{
Wave Propagation in a Medium with Disordered Excitability
}

\author{
I. Sendiña-Nadal, ${ }^{1, *}$ A. P. Muñuzuri, ${ }^{1}$ D. Vives,${ }^{2}$ V. Pérez-Muñuzuri, ${ }^{1}$ J. Casademunt,${ }^{3}$ L. Ramírez-Piscina, ${ }^{4}$ \\ J. M. Sancho, ${ }^{5, \dagger}$ and F. Sagués ${ }^{2}$ \\ ${ }^{1}$ Group of Nonlinear Physics, Faculty of Physics, University of Santiago de Compostela, 15706 Santiago de Compostela, Spain \\ ${ }^{2}$ Departament de Química Física, Universitat de Barcelona, Diagonal 647, 08028 Barcelona, Spain \\ ${ }^{3}$ Departament d'Estructura i Constituents de la Matèria, Universitat de Barcelona, Diagonal 647, 08028 Barcelona, Spain \\ ${ }^{4}$ Departament de Física Aplicada, Universitat Politècnica de Catalunya, Gregorio Marañón 44, 08028 Barcelona, Spain \\ ${ }^{5}$ Institute for Nonlinear Science, University of California at San Diego, 9500 Gilman Drive, \\ Department 0402, La Jolla, California 92093-0402
}

(Received 24 October 1997; revised manuscript received 23 February 1998)

\begin{abstract}
The effect of quenched disorder on the propagation of autowaves in excitable media is studied both experimentally and numerically in relation to the light-sensitive Belousov-Zhabotinsky reaction. The spatial disorder is introduced through a random distribution with two different levels of transmittance. In one dimension the (time-averaged) wave speed is smaller than the corresponding to a homogeneous medium with the mean excitability. Contrarily, in two dimensions the velocity increases due to the roughening of the front. Results are interpreted using kinematic and scaling arguments. In particular, for $d=2$ we verify a theoretical prediction of a power-law dependence for the relative change of the propagation speed on the disorder amplitude. [S0031-9007(98)06327-3]
\end{abstract}

PACS numbers: 82.40.Bj, 03.40.Kf, 47.54. $+\mathrm{r}$

Wave propagation in homogeneous excitable media has been studied extensively in the past years [1]. Representative examples are spiral and target waves in the BelousovZhabotinsky (BZ) reaction [2], reentry formation in excitable cardiac tissues, or spiral-like organization of the slime mold Dictiostelium discoideum [3].

Propagation of excitable waves in inhomogeneous media has also been studied from different points of view, such as the interaction of waves and inert obstacles (which is of some interest in cardiology, since reentries can be anchored [4-6] or planar fronts broken [7-10]) and the effect of modulations [11,12] or fluctuations [13-15]. Besides, the influence of additive and multiplicative fluctuations on symmetric bistable wave motion has been analyzed in detail [16-19].

On the other hand, front roughness induced by fluctuations has received recently increasing attention [20,21]. Particularly analyzed have been the quenched versions [22,23] of either the Edwards-Wilkinson [24] or the Kardar-Parisi-Zhang (KPZ) equations [25] as they apply to kinetic roughening induced by time-independent disorder.

In this Letter we aim at studying, both experimentally and numerically, the effects on the propagation of autowaves originated by introducing time-independent random spatial fluctuations in the medium excitability.

Experimentally a photosensitive highly excitable BZ medium was chosen. As shown in Fig. 1, we have considered two distinct configurations, where two parts of the medium, the leftmost homogeneous and the rightmost inhomogeneous, were separated by a vertical, completely unexcitable, strip with higher illumination. In the quasione-dimensional configuration, horizontal stripes of random dichotomic illumination, with the same average light intensity as in the homogeneous part, introduced the disorder. The two-dimensional setup was prepared analogously, this time with randomly distributed squares of two possible light intensities. The typical experiments (see Fig. 1) consisted in generating a planar wave at the bottom of the medium and observing its upwards evolution along the vertical axis. In this way both the shapes and velocities of the two free-end noninteracting
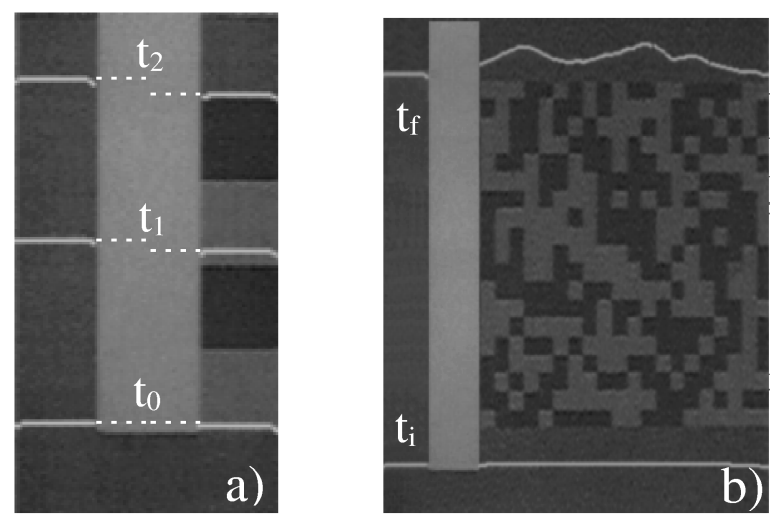

FIG. 1. Propagating wave fronts on light-sensitive media consisting of both a left-side homogeneous and a right-side inhomogeneous medium with a brighter strip between them where fronts cannot propagate. (a) Quasi-one-dimensional setup. An initial flat front splits into two that were represented at three different times. The front which propagates through the inhomogeneous part undergoes an appreciable delay with respect to the other one. Stripe width in the direction of propagation $l=1.1 \mathrm{~cm}$. (b) Two-dimensional setup with randomly distributed squares. An initial flat front gets rough in the randomly illuminated zone and goes faster than in the homogeneous part; square size $l=2.3 \mathrm{~mm}$ and size of the medium in the transversal direction to the propagation $5.4 \mathrm{~cm}$. 
fronts at either side of the central strip were compared. Experiments were performed with the BZ reagent catalyzed by the ruthenium bipyridyl complex $\mathrm{Ru}(\mathrm{bpy})$ which is sensitive to visible light [26-29]. The Ru complex was immobilized in a silica-gel matrix [using a solution of $15 \%$ sodium silicate, $0.71 \mathrm{mM} \mathrm{Ru(bpy})_{3}^{2+}$ and $0.18 \mathrm{M}$ $\mathrm{H}_{2} \mathrm{SO}_{4}$; preparation as in [30]] in a Petri dish (diameter, $9.6 \mathrm{~cm}$; thickness, $1 \mathrm{~mm}$ ). The solution (initial concentrations: $0.18 \mathrm{M} \mathrm{KBr}, 0.33 \mathrm{M}$ malonic acid, $0.39 \mathrm{M} \mathrm{NaBrO}_{3}$ and $0.69 \mathrm{M} \mathrm{H}_{2} \mathrm{SO}_{4}$ ) was pored onto the gel. The temperature was kept constant at $25 \pm 1{ }^{\circ} \mathrm{C}$. White light $(190 \mathrm{~W}$ halogen lamp) passed first a diffusion screen, then the Petri dish, the interference filter $(450.6 \mathrm{~nm}$; transmission $56 \%$ ), and finally video equipment for image recording $[31,32]$. In order to prepare the nonuniform excitability on the gel, a transparency was printed with a pattern of transmittances, in such a way that when it is placed between the light source and the Petri dish the light distribution described above is projected onto the gel. In the inhomogeneous part, the transparency had a transmittance $T$ given by

$$
T(x, y)=T_{0}+\delta T \eta(x, y),
$$

where $x$ is the transversal direction to front propagation, $T_{0}$ is the mean transmittance, and $\eta(x, y)= \pm 1$ is a twovalued random number of zero spatial average. Within the illumination range here considered, speeds of planar autowaves decrease linearly with light intensity [11].

Typical experimental observations are shown in Fig. 1. In the quasi-1D arrangement [Fig. 1(a)], planar fronts in the inhomogeneous part are observed to go slower on average than those propagating under uniform illumination corresponding to the spatial average of the inhomogeneous region. Contrarily, in 2D [Fig. 1(b)], dynamically evolving, distorted although still well-defined autowaves propagate faster.

Numerically, we conducted simulations with a twovariable Oregonator model adapted to our photosensitive medium [33],

$$
\begin{aligned}
\frac{\partial u}{\partial t}= & \frac{1}{\varepsilon}\left(u-u^{2}-[f g+\phi(x, y)] \frac{u-q}{u+q}\right) \\
& +D_{u} \nabla^{2} u \\
\frac{\partial g}{\partial t}= & (u-g)+D_{g} \nabla^{2} g,
\end{aligned}
$$

where $u(g)$ describes $\mathrm{HBrO}_{2}$ (catalyst) concentrations. $D_{u}$ and $D_{g}$ are diffusion coefficients with $D_{g}=0$ (to reproduce that the catalyst is immobilized). $f, q$, and $\varepsilon$ are parameters related to the kinetics of the $\mathrm{BZ}$ reaction. $\phi(x, y)$ represents the light-induced flow of $\mathrm{Br}^{-}$and it is assumed directly proportional to $T(x, y)$. Since edge effects at the boundaries of the nonexcitable central strip are negligible, simulations have been performed with lateral periodic boundary conditions for simplicity.

Before proceeding further let us clearly state the conditions with which our experimental and numerical study complies. This will enable us to interpret the observed results in terms of generic kinematic arguments widening in this way the scope of our study beyond the particular randomness realization here analyzed. Listing them separately: (i) We restrict ourselves to thin fronts measured on the length scale introduced by the disorder. (ii) We verify, both experimentally and numerically, that the autowave speed adapts quasiadiabatically to the local illuminating conditions. (iii) Finally, in the 2D case, we assume that the disorder amplitude, i.e., a measure of the dispersion of local velocities in our two-state model is small.

In particular, for the 2D case, the above conditions lead us to conclude that diffusion lengths in this problem will always be considerably smaller than the length scale associated with the front deformation. In turn this justifies the use, both as a central theoretical issue as well as an advantageous computational resource, of the linear speedcurvature relation, also well-known as the first-order eikonal equation [34], which gives the normal velocity of the autowave in terms of its local plane-wave value corrected by a curvature term. On the other hand, by invoking condition (ii), this local velocity is assumed to be at any time fixed by the space-dependent illumination. We translate such a relation into Cartesian coordinates for the position of the front, denoted $y=h(x, t)$, to have

$$
h_{t}=v(x, h) \sqrt{1+\left(h_{x}\right)^{2}}+D \frac{h_{x x}}{1+\left(h_{x}\right)^{2}},
$$

where $D$ is an effective diffusion coefficient and, in general, a function of diffusion coefficients of the species involved in the front propagation. This coefficient is assumed to be independent of the light intensity $[11,35]$. Besides conditions (i) and (iii) and the assumption of isolated fronts, allow us to consider Eq. (3) valid within the Zykov's limit (see Ref. [34]). D has been estimated from the integration of the full Oregonator model with circular fronts. To validate our assumptions, the results of both versions, the complete reaction-diffusion scheme (2) and the local one (3), were checked to give the same results with great accuracy, for both front shapes and velocities.

Let us formulate our theoretical scenario. In a 1D situation, and invoking conditions (i) and (ii) above, the propagating interface can be viewed as a pointlike object which follows instantaneously a spatial profile of velocity $v(y)$. It can be proved that for an arbitrary (non-negative) velocity profile $v(y)$ the following inequality holds

$$
\bar{v}=L\left[\int_{0}^{L} v(y)^{-1} d y\right]^{-1} \leq L^{-1} \int_{0}^{L} v(y) d y
$$

with the equality holding for the homogeneous case. We thus conclude that the time-averaged velocity is always smaller than its spatial average. If $v(y)$ is a random variable, the previous statement is valid for each realization of it. However, it may be convenient to relate $\bar{v}$ to the statistical properties of $v(y)$. If $L$ is large enough 
compared to the spatial correlations of the disorder and under self-averaging conditions, the integral over $L$ in the left part of Eq. (4) is equivalent to an ensemble average in each point, that is,

$$
\frac{1}{\bar{v}}=\left\langle\frac{1}{v(y)}\right\rangle .
$$

If we write $v(y)=v_{0}+\delta v(y)$ with $\delta v(y)$ having a vanishing spatial average, and being bounded by $|\delta v(y)|<v_{0}$, such an expression can be expanded as

$$
\frac{1}{\bar{v}}=\frac{1}{v_{0}} \sum_{n=0}^{\infty}(-1)^{n}\left\langle\left(\frac{\delta v}{v_{0}}\right)^{n}\right\rangle,
$$

which relates the time-averaged velocity to the statistical moments of the disorder. For the two-state model, $\delta v \equiv$ $\pm \Delta v$, the previous expression can be computed exactly giving a reduced velocity

$$
\frac{\bar{v}-v_{0}}{v_{0}}=-\left(\frac{\Delta v}{v_{0}}\right)^{2} .
$$

In $2 \mathrm{D}$ the situation is a bit more involved. We start directly from our central equation (3) and develop it, consistently with approximations (i)-(iii) above, using a small-gradient approximation. Noting $v(x, h)=v_{0}+$ $\delta v(x, h)$ and retaining as usual the lowest-order nonlinearity on $h_{x}$ we have

$$
h_{t}=v_{0}+\frac{v_{0}}{2}\left(h_{x}\right)^{2}+D h_{x x}+\delta v(x, h),
$$

where an extra multiplicative term $\left(h_{x}\right)^{2} \delta v(x, h)$ has been neglected for weak enough disorder amplitudes. Notice that, written in this way and after some trivial reparametrization to get rid of the trivial $v_{0}$ term, this equation strongly resembles the well-known KPZ model

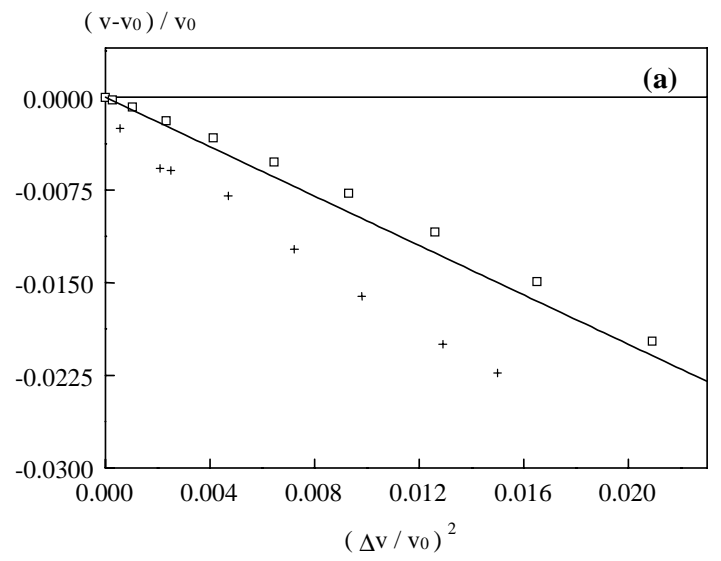

for the propagation of random interfaces [25]. A quite similar KPZ-like equation was also considered by Kerstein and Ashurst [36] when dealing with interfaces propagating in randomly advected media. The only difference is that the diffusive term $h_{x x}$, responsible for the transverse redistribution of fluctuations along the interface, is there replaced by a similar term on $h_{x}$ times the transverse component of the advecting flow. Furthermore, they selfconsistently showed that for weak randomness (weak stirring in their language) such transverse redistribution effect is irrelevant for their scaling analysis. What makes such an analysis particularly appropriate to our situation is that they focused explicitly on the limit of frozen flows, which is completely equivalent to the scenario of quenched disorder here addressed. Under these conditions their main result reads [36]

$$
S=1+\beta Q^{4 / 3},
$$

where $S \equiv v / v_{0}$ and $Q$ represent a relative measure of the randomness in the medium. In our notation $Q \equiv$ $|\Delta v| / v_{0}$, so Eq. (9) transforms into

$$
\frac{\bar{v}-v_{0}}{v_{0}}=\beta\left[\left(\frac{\Delta v}{v_{0}}\right)^{2}\right]^{2 / 3} \text {. }
$$

The theoretical predictions, represented, respectively, by Eqs. (7) and (10), are compared with numerical and experimental results in Fig. 2. Certainly, the quantitative agreement is extremely rewarding with respect to the numerical simulations. In particular, the observed power laws and corresponding exponent are certainly consistent with the theoretical prediction, whereas the prefactor $\beta$ in Eq. (10) is clearly seen to depend on the length scale of the spatial inhomogeneities. Experimental results

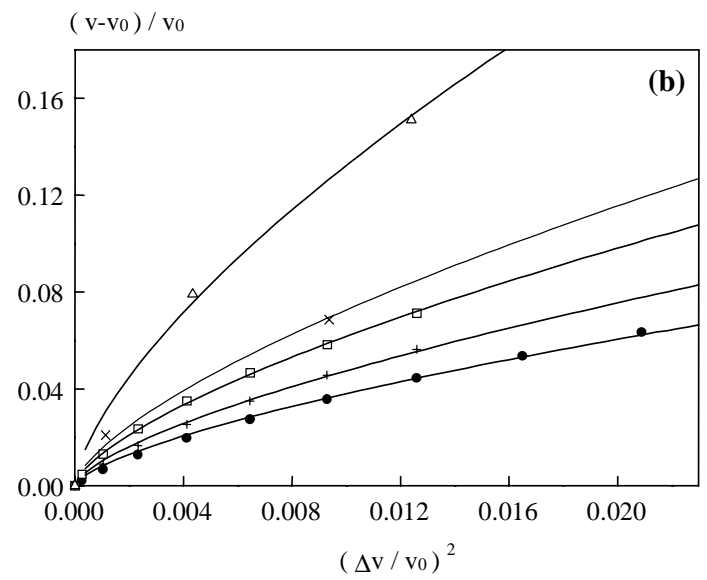

FIG. 2. Dependence of the relative variation in wave velocity vs amplitude disorder both numerically $\left(\phi_{0}=0.01\right)$ and experimentally $\left(T_{0}=3.55 \mathrm{~W} / \mathrm{m}^{2}\right)$. (a) One-dimensional system. Continuous line (slope -1 ) represents the theoretical prediction of Eq. (7). Numerical points ( $\square$ ) were simulated with stripes of width 10 s.u. (space units) and the best linear fit corresponds to a slope of -0.93 ; experimental data $(+)$ were obtained with squares of length of $1.1 \mathrm{~cm}$ and adjusted to a line of slope -1.42 . (b) Two-dimensional system. Numerically: for a medium width of 160 s.u. and noise squares of side length $10(\bullet), 20(+)$, and 40 ( $\square$ ). Continuous lines represent nonlinear fits of Eq. (10), with $\beta=0.831,1.04$, and 1.35, respectively. The typical front width is about $2.5 \mathrm{s.u}$. Experimental results shown for a medium width of $5.4 \mathrm{~cm}$ and $l=2.3 \mathrm{~mm}(\times)$, and for $6 \mathrm{~cm}$ and $l=2.7 \mathrm{~mm}$ $(\triangle)$, with $\beta=1.59$ and 2.89, respectively. Model parameters: $f=3, q=0.002, \varepsilon=0.05, D_{u}=1, D_{g}=0$, and $\phi_{0}=0.01$. 
also support the previous theoretical considerations given the unavoidable experimental limitations mainly due to light dispersion and intrinsic inaccuracies in velocity measurements. This effect is shown in Fig. 2(a) where experimental data deviate from the theoretical prediction (continuous line) as the amplitude of disorder increases. Here, the inhibitor which is continuously produced in the brighter squares tends to invade the darker ones, then increases the effective value of the transmittance there. This effect becomes more important as the amplitude of the disorder increases. Therefore, the corresponding mean value of the transmittance $T_{0}$ in Eq. (1) varies slightly with the disorder amplitude. In any case, the distinctive behaviors depending on the dimension considered are clearly exhibited in the experiments.

Special thanks to Dr. Gómez-Gesteira for his useful advice about experimental aspects. D. V. benefited from a FI grant from Generalitat de Catalunya. This work was partially supported by the Comisión Interministerial de Ciencia y Tecnología (Spain) under Projects No. DGICYT-PB94-0623, No. PB96-1001, No. PB96-0241, and No. PB96-0937 and Comissionat per a Universitats i Recerca (Generalitat de Catalunya). Calculations were performed at the Supercomputation Centers of Catalunya (CESCA) and Galicia (CESGA), Spain.

*Corresponding author.

Email address:irene@fmmeteo.usc.es

†Permanent address: Departament d'Estructura i Constituents de la Matèria, Universitat de Barcelona, Diagonal 647, 08028 Barcelona, Spain.

[1] J. J. Tyson and J.P. Keener, Physica (Amsterdam) 32D, 327 (1988); E. Meron, Phys. Rep. 218, 1 (1992).

[2] A.N. Zaikin and A.M. Zhabotinsky, Nature (London) 225, 535 (1970).

[3] N. A. Robertson and A. F. Grutsch, Cell 24, 603 (1983).

[4] G. R. Mines, Trans. R. Soc. Can. 4, 43 (1914).

[5] M. A. Allesie, F. M. I. Bonke, and T. Y. G. Scopman, Circ. Res. 33, 54 (1973).

[6] M.S. Spach, W. T. Miller, D. B. Geselowitz, R. C. Borr, J. M. Kootsey, and E. A. Johnson, Circ. Res. 48, 39 (1981).

[7] A. T. Winfree, J. Theor. Biol. 40, 45 (1973).

[8] A. Karma, Phys. Rev. Lett. 71, 1103 (1993).

[9] M. Gómez-Gesteira, J.L. del Castillo, M. E. VázquezIglesias, V. Pérez-Muñuzuri, and V. Pérez-Villar, Phys. Rev. E 50, 4646 (1994).

[10] J. M. Starobin and C.F. Starmer, Phys. Rev. E 54, 430 (1996).

[11] I. Sendiña-Nadal, M. Gómez-Gesteira, V. PérezMuñuzuri, V. Pérez-Villar, J. Armero, L. Ramírez-Piscina, J. Casademunt, F. Sagués, and J. M. Sancho, Phys. Rev. E 56, 6298 (1997).
[12] J. Armero, A. M. Lacasta, L. Ramírez-Piscina, J. Casademunt, J. M. Sancho, and F. Sagués, Europhys. Lett., 33, 429 (1996); Phys. Rev. E, 56, 5405 (1997).

[13] P. Jung and G. Mayer-Kress, Phys. Rev. Lett. 74, 2130 (1995); Chaos 5, 458 (1995).

[14] P. Jung, Phys. Rep. 234, 175 (1993).

[15] J. Maselko and K. Showalter, Physica (Amsterdam) 49D, 21 (1991).

[16] A.S. Mikhailov, L. Schimansky-Geier, and W. Ebeling, Phys. Lett. 96A, 453 (1983).

[17] V. Pérez-Muñuzuri, M. Gómez-Gesteira, V. Pérez-Villar, and L. O. Chua, Int. J. Bif. Chaos 3, 211 (1993).

[18] H. P. Breuer, W. Huber, and F. Petruccione, Physica (Amsterdam) 73D, 259 (1994).

[19] J. Armero, J. M. Sancho, J. Casademunt, A. M. Lacasta, L. Ramírez-Piscina, and F. Sagués, Phys. Rev. Lett. 76, 3045 (1996).

[20] Dynamics of Fractal Surfaces, edited by F. Family and T. Vicsek (World Scientific, Singapore, 1991)

[21] A.-L. Barabasi and H.E. Stanley, Fractal Concepts in Surface Growth (Cambridge University Press, Cambridge, England, 1995).

[22] T. Natterman, S. Stepanow, L.-H. Tang, and H. Leschhorn, J. Phys. (Paris) II 2, 1483 (1992).

[23] L. A. N. Amaral, A.-L. Barabasi, and H. E. Stanley, Phys. Rev. Lett. 73, 62 (1994).

[24] S. F. Edwards and D. R. Wilkinson, Proc. R. Soc. London A 381, 17 (1982).

[25] M. Kardar, G. Parisi, and Y.C. Zhang, Phys. Rev. Lett. 56, 889 (1986).

[26] L. Kuhnert, Nature (London) 319, 393 (1986).

[27] L. Kuhnert, K. I. Agladze, and V.I. Krinsky, Nature (London) 337, 224 (1989).

[28] M. Markus, Zs. Nagy-Ungvaray, and B. Hess, Science 257, 225 (1992).

[29] O. Steinbock, V.S. Zykov, and S.C. Müller, Nature (London) 366, 322 (1993).

[30] T. Yamaguchi, L. Kuhnert, Zs. Nagy-Ungvaray, S. C. Müller, and B. Hess, J. Phys. Chem. 95, 5831 (1991).

[31] A.P. Muñuzuri, V.A. Davydov, M. Gómez-Gesteira, V. Pérez-Muñuzuri, and V. Pérez-Villar, Phys. Rev. E 54, R5921 (1996).

[32] A.P. Muñuzuri and M. Markus, Phys. Rev. E 55, 33 (1997).

[33] H.-J. Krug, L. Pohlmann, and L. Kuhnert, J. Phys. Chem. 94, 4862 (1990).

[34] V.S. Zykov, Biofizika 25, 888 (1980) [Biophysics 25, 906 (1980)]. For a much more recent treatment, see also A. M. Pertsov, M. Wellner, and J. Jalife, Phys. Rev. Lett. 78, 2656 (1997); M. Wellner and A. M. Pertsov, Phys. Rev. E 55, 7656 (1997), and references therein.

[35] V. Petrov, Q. Ouyang, G. Li, and H. L. Swinney, J. Phys. Chem. 100, 18992 (1996).

[36] A. R. Kerstein and Wm. T. Ashurst, Phys. Rev. Lett. 68, 934 (1992). 